\title{
Laparoscopic Management of a Canal of Nuck Cyst
}

\author{
Jacqueline Ho, MD, John Maa, MD, Peter Liou, MD, Jeannette Lager, MD \\ Department of Obstetrics and Gynecology, and Reproductive Sciences, University of California San Francisco, CA \\ (Drs. Ho, Lager). \\ Northern California Chapter of the American College of Surgeons, and Division of General and Trauma Surgery, Marin \\ General Hospital, Larkspur, CA (Dr. Maa). \\ Department of Surgery, Columbia University, New York, NY (Dr. Liou).
}

\begin{abstract}
The female hydrocele, also known as the canal of Nuck cyst, is a rare congenital abnormality that is the equivalent of the patent processus vaginalis in males. We are the first to report the laparoscopic excision of an entirely extraperitoneal canal of Nuck cyst. We discuss the embryology, pathophysiology, and surgical management of this atypical variant of a rare entity.
\end{abstract}

Key Words: Canal of Nuck cyst, Hydrocele, Laparoscopic surgery, Processus vaginalis.

\footnotetext{
Citation Ho J, Maa J, Liou P, Lager J. Laparoscopic management of a canal of nuck cyst. CRSLS e2014.002134. DOI: 10.4293/CRSLS.2014.002134.

Copyright (c) 2014 SLS This is an open-access article distributed under the terms of the Creative Commons Attribution-Noncommercial-ShareAlike 3.0 Unported license, which permits unrestricted noncommercial use, distribution, and reproduction in any medium, provided the original author and source are credited.

Presented at the Abdominal Wall Reconstruction Conference, June 12-14, 2014, Washington, DC.

Address correspondence to: John Maa, MD, Marin General Hospital, 5 Bon Air Road \#101, Larkspur, CA 94939. E-mail: maaj@maringeneral.org
}

\section{INTRODUCTION}

The canal of Nuck cyst is a rare congenital abnormality resulting from a partial or incomplete obliteration of the female counterpart of the processus vaginalis, which carries the round ligament. ${ }^{1}$ Typically, the processus vaginalis self-obliterates within the first year of life. Failure of this to happen may result in either an inguinal hernia or a communicating hydrocele. We describe an atypical case of a canal of Nuck cyst that was successfully resected laparoscopically, without the need for an open-groin incision. This case was unique in that the internal inguinal ring was not patent, and the cyst was located in the extraperitoneal space of Retzius, an analogue to a noncommunicating hydrocele in a man.

\section{CASE REPORT}

A 36-year-old woman, gravida 1 para 0 , initially presented to the general surgery service with a bulge in her right lower quadrant that had expanded progressively over the previous several months and was thought to be an inguinal hernia by her primary care provider. She had a history of breast cancer treated with partial mastectomy and adjuvant chemoradiation. Her symptoms of fullness and discomfort worsened with standing and improved with lying supine. On exami- nation, she had a palpable 5-cm cystic fluid collection lateral to the pubis in the region of her right inguinal canal.

A computed tomography (CT) scan obtained by her referring provider revealed a fluid collection in the right anterior pelvis that had increased in size from 9 months earlier and was thought to represent a lymphocele, seroma, ovarian cyst, or other embryologic remnant of Müllerian duct origin (Figure 1). The patient was referred to the urology service because of the collection's proximity to the bladder and the possible diagnosis of a hydrocele. A cystoscopy with cystogram was obtained and showed no evidence of bladder diverticulum. Fine-needle aspiration of the cyst drained $30 \mathrm{~mL}$ of serosanguineous fluid, which promptly reaccumulated. The fluid creatinine level was low, suggesting that this collection was not urinary in origin. Cytologic examination showed mature lymphocytes and macrophages, without evidence of carcinoma. The collection was thought likely to be a female hydrocele, or canal of Nuck cyst. The patient was then referred to the gynecology service for further treatment.

The patient declined initial surgical management because she became pregnant, but after having a spontaneous abortion, she chose to undergo surgical repair to optimize the success of a future pregnancy. Her symptoms from the 


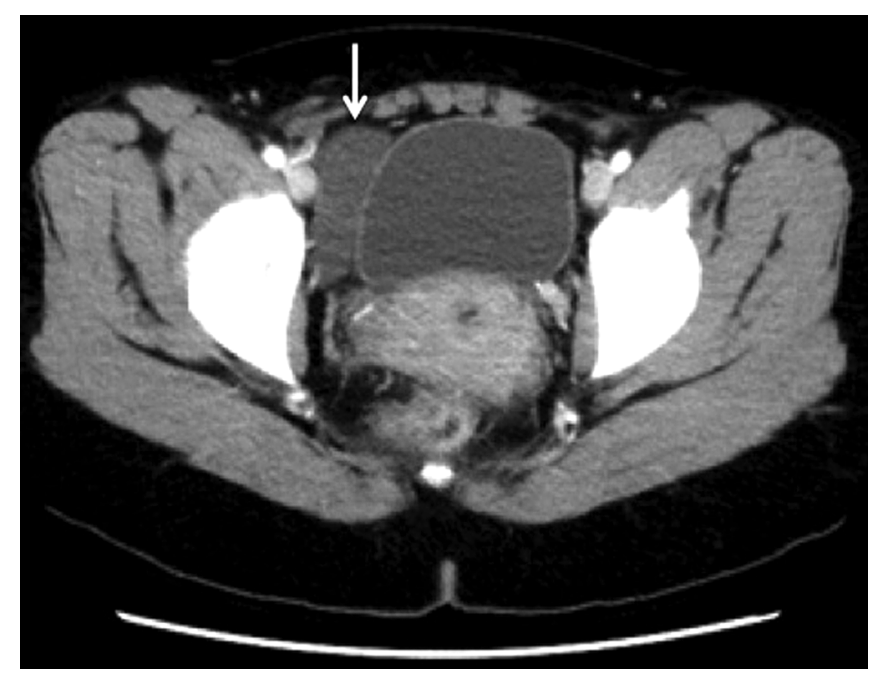

Figure 1. An axial image from the CT scan demonstrating a fluid collection in the right anterior pelvis adjacent to the bladder.

cyst worsened throughout the day as the cyst increased in size, and there was concern that her discomfort could worsen during pregnancy as a result of the pressure from the gravid uterus. At repeat evaluation, pelvic ultrasonography demonstrated a $5 \times 5-\mathrm{cm}$ anechoic subcutaneous fluid collection thought most likely to be a canal of Nuck hydrocele (Figure 2). However, there was concern of adjacent bowel contents entering the superior neck of the cyst when a Valsalva maneuver was performed, raising the possible diagnosis of a concomitant inguinal or femoral hernia. A small fibroid was noted, but the fallopian tubes and ovaries appeared normal.

A magnetic resonance imaging (MRI) scan was obtained, revealing that the small amount of loculated fluid in the

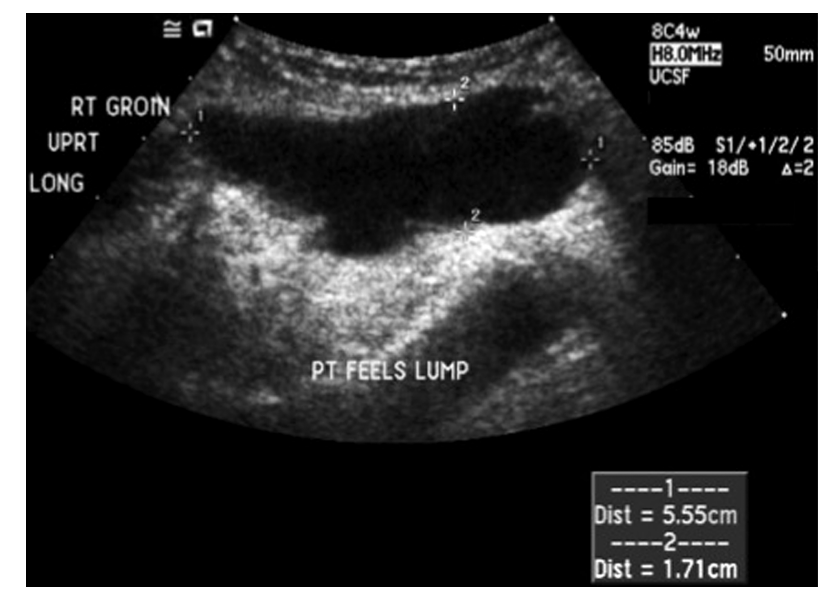

Figure 2. Pelvic ultrasonography demonstrating a $5.6 \times 1.7-\mathrm{cm}$ anechoic subcutaneous fluid collection. right lower pelvis to the right of the bladder had increased compared with prior imaging (Figure 3). The fluid approached the right inguinal canal but did not enter it. There was a small channel with trace fluid within the right inguinal region, consistent with a canal of Nuck. Of note, there was no evidence of metastatic disease from her history of breast carcinoma. The repeat CT scan confirmed that the pelvic fluid collection of $6.4 \times 1.5 \mathrm{~cm}$ was separate from the bladder or ovary and thought to be possibly of lymphatic origin.

Given the uncertain diagnosis and challenging location of the cyst, the patient underwent surgery performed by a team consisting of a gynecologist, general surgeon, and urologist. A laparoscopic approach was initially planned, with the option to utilize a kidney transplant incision, if necessary, to access the cyst. Laparoscopy revealed a mild dimpling of the right internal ring but no evidence of an inguinal hernia (Figure 4a). Most of an extraperitoneal cyst located in the space of Retzius was removed laparoscopically with a combination of sharp and blunt dissection. The peritoneum was incised and a cyst excision performed, with dissection of the tissue adjacent to the bladder away from the cyst (Figure $4[\mathbf{B}]$ ). A mesh reconstruction of the inguinal floor was not performed because a hernia was not identified. Pelvic washings obtained because of the patient's history of breast cancer were

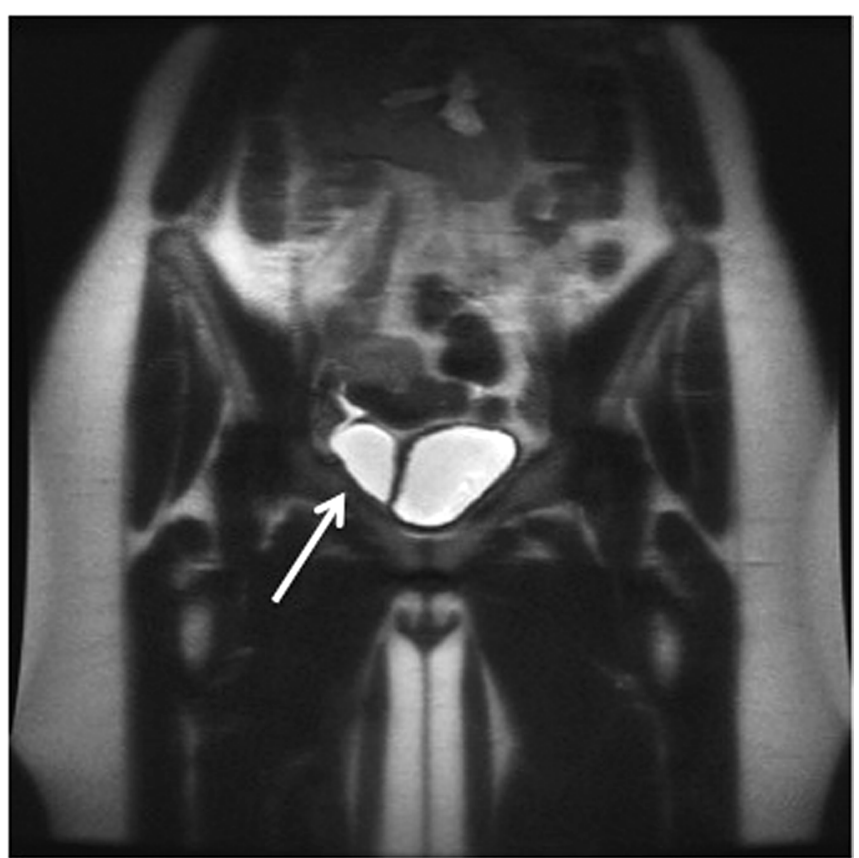

Figure 3. Magnetic resonance imaging (MRI) demonstrating a loculated fluid collection in the pelvis to the right of the bladder. 

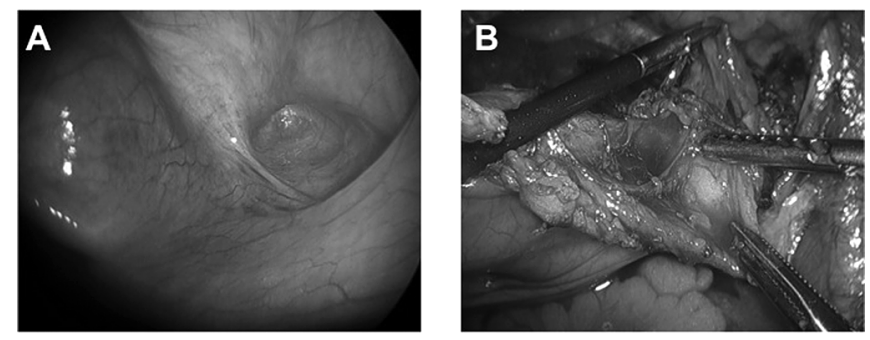

Figure 4. A, Intraoperative photograph during laparoscopic surgery demonstrating a dimpling of the right internal ring but no evidence of an inguinal hernia. B, Intraoperative photograph after the peritoneum was incised and during cyst excision.

negative. Pathologic analysis of the cyst wall showed benign mesothelium and fibroadipose tissue with chronic inflammation, consistent with a canal of Nuck cyst. The patient had an uncomplicated recovery, and at 1-year follow-up, the cyst had not recurred.

\section{DISCUSSION}

The canal of Nuck is a rare congenital abnormality resulting from a partial or incomplete obliteration of the female counterpart of the processus vaginalis, which carries the round ligament. ${ }^{2}$ Normal embryologic development of the reproductive and urinary organs includes a descent of the gubernaculum into the processus vaginalis. The gubernaculum is undifferentiated mesenchyme attached to the caudal aspect of the gonads to guide them in descent. In males, this involves the testicles passing through the internal inguinal ring into the scrotum. In females, the peritoneum evaginates and obliterates proximally at the inguinal ring. However, in rare instances, incomplete obliteration of the canal of Nuck, along with hypersecretion, incomplete reabsorption, and other idiopathic mechanisms, leads to collection of fluid in the canal. ${ }^{3}$

Although it is not harmful, a canal of Nuck cyst can cause discomfort and pain. There are rare instances of abnormal pathology involving the canal of Nuck, including endometriosis, infection, and neoplasm.4,5 However, most cases are benign. The differential diagnosis includes an inguinofemoral hernia, lymphadenopathy, Bartholin cyst, vascular malformations, bony structures, and malignant or benign tumors. ${ }^{6}$

The term canal of Nuck cyst is often used by radiologists to describe a fluid-filled mass in a woman's groin that disappears while she is lying supine and reaccumulates upon her assuming a standing position. These cysts can be diagnosed by high-resolution ultrasonography that shows a typical appearance of a circumscribed cystic mass, with occasional septae and contiguity with the round ligament. ${ }^{7}$ A sonographic anechoic cystic structure as well as hyperintensity on T2-weighted MRI support a diagnosis of a cystic mass, which is consistent with prior imaging reports of canal of Nuck cysts. ${ }^{7,8}$ Sometimes fluid can be seen extended to a neck of the cyst with external compression by the ultrasound probe, or during a Valsalva maneuver to elicit a concomitant hernia.?

Traditionally, canal of Nuck cysts have been repaired through an open approach. Most previous reports describe a groin approach for excision of the mass, but laparoscopy has been used to diagnose canal of Nuck cysts, ${ }^{10}$ and a recent case describes the ligation of a patent canal of Nuck when the cyst is diagnosed incidental to another laparoscopic procedure. ${ }^{11}$ Approximately 30\% to $40 \%$ of these cysts are associated with concomitant inguinal hernias. ${ }^{2}$ A laparoscopic approach has not been previously used to excise these cysts. In the case of a concomitant inguinal hernia, a key advantage of laparoscopy is the ability to evaluate the contralateral side for hernia. ${ }^{12}$ In our case, it was beneficial to perform laparoscopy because the diagnosis was initially uncertain.

Very few published reports describe the entirely laparoscopic management of canal of Nuck cysts. ${ }^{10,11}$ An entirely extraperitoneal location such as the one in our patient is atypical and can be difficult to definitively diagnose on preoperative imaging, where the finding may mimic a duplication of the bladder or an inguinal hernia. In the situation where the etiology of a female groin mass is uncertain, direct visualization with laparoscopy can be particularly helpful. The urology service provided expertise for the safe dissection in this space, because they are accustomed to working in the space of Retzius for robotic prostatectomy. Both the laparoscopic and open approaches to the surgical management of canal of Nuck cysts are safe, and patients can generally be discharged home after same-day surgery.

In our patient, CT of the abdomen and pelvis was not suggestive of the diagnosis of an inguinal hernia because the fluid had not entered the inguinal canal or extended medially to the pubic tubercle. The radiographic findings in this case were unusual in that the cyst was not located in the subcutaneous tissue, but rather it was adjacent to the bladder in the pelvis, which initially led us to believe this may have been a cyst of urologic origin. Findings at surgery did not demonstrate any communication with the peritoneal cavity. The cyst was located in the space of Retzius and was entirely extraperitoneal. This case was atypical because the internal inguinal ring was not patent, 
indicating that it was a variant of a noncommunicating hydrocele in a man.

\section{CONCLUSION}

The treatment of an atypical presentation of a canal of Nuck hydrocele in the space of Retzius can be achieved through an entirely laparoscopic approach. A traditional open approach would have been difficult because excision requires disruption of the floor of the inguinal canal. A kidney transplant incision can be used if necessary, but avoiding disruption of the inguinal floor is preferable. We conclude that laparoscopy is a safe way to aid in the diagnosis and treatment for canal of Nuck cysts, especially when diagnostic imaging is inconclusive.

The authors thank Drs. Peter Carroll and James Smith of the Urology Service for their expertise in the intraoperative approach, and to Pamela Derish for writing assistance.

\section{References:}

1. Counseller VS, Black BM. Hydrocele of the canal of Nuck: report of seventeen Cases. Ann Surg. 1941;113(4):625-630.

2. Caviezel A, Montet X, Schwartz J, et al. Female hydrocele: the cyst of Nuck. Urologia internationalis. 2009;82(2):242-245.

3. Jagdale R, Agrawal S, Chhabra S, Jewan SY. Hydrocele of the canal of Nuck: value of radiological diagnosis. J Radiol Case Rep. 2012;6(6):18-22.
4. Cervini P, Wu L, Shenker R, et al. Endometriosis in the canal of Nuck: atypical manifestations in an unusual location. Can J Plast Surg. 2004;12(2):73-75.

5. Wang CJ, Chao AS, Wang TH, et al. Challenge in the management of endometriosis in the canal of Nuck. Fertil Steril. 2009;91(3):936;e939-911.

6. Bunni J, Gillam M, Pope IM. Hydrocele of the canal of Nuck-an old problem revisited. Front. Med. 2013;7(4):517-519.

7. Stickel WH, Manner M. Female hydrocele (cyst of the canal of Nuck): sonographic appearance of a rare and little-known disorder. J Ultrasound Med. 2004;23(3):429-432.

8. Safak AA, Erdogmus B, Yazici B, Gokgoz AT. Hydrocele of the canal of Nuck: sonographic and MRI appearances. J Clin Ultrasound. 2007;35(9):531-532.

9. Yigit H, Tuncbilek I, Fitoz S, et al. Cyst of the canal of Nuck with demonstration of the proximal canal: the role of the compression technique in sonographic diagnosis. J Ultrasound Med. 2006;25(1):123-125.

10. Bunting D, Szczebiot L, Cota A. Laparoscopic hernia repairwhen is a hernia not a hernia? JSLS. 2013;17(4):654-656.

11. Yen CF, Wang CJ, Chang PC, et al. Concomitant closure of patent canal of Nuck during laparoscopic surgery: case report. Human Reprod. 2001;16(2):357-359.

12. Lau ST, Lee YH, Caty MG Current management of hernias and hydroceles. Semin Pediatr Surg. 2007;16(1):50-57. 